\title{
High Pressure Freezing and Freeze Substitution for Immunolabeling in a Core Imaging Facility
}

\author{
J.S. Strong, ${ }^{*}$ J.E. Goolsby, ${ }^{*}$ and R.-c. Hsia* \\ * Core Imaging Facility, University of Maryland Dental School, 650 West Baltimore Street, \\ Baltimore, MD 21201
}

Immuno electron microscopy (ImmunoEM) is a vital technique to study localization and interaction of protein molecules at the cellular level. The most commonly used immuno EM methods include pre-embedding and post-embedding immunolabeling using gold-conjugated antibodies. The postembedding method involves embedding samples in a hydrophilic acrylic resin and performing immunogold labeling on ultrathin sectioned specimens on TEM grids. This method is less time consuming and allows multiple labelings of the same specimen with a variety of antibodies and antibody dilutions. A specimen that is well preserved in acrylic resin can be sectioned repetitively over several years to generate grids for immunolabeling. However, in order to preserve antigenicity, samples for post embedding immunolabeling must be processed with minimum chemical fixation such that preservation of the ultrastructure may be compromised when compared to that of EM specimens prepared using traditional combinations of chemical fixatives, e.g. paraformaldehyde, glutaraldehyde and osmium tetroxide. Cryo-fixation using rapid cooling under high pressure followed by freeze substitution has been shown to provide a suitable alternative that bypasses problems associated with chemical fixation while preserving ultrastructural and antigenic integrity for the purpose of immunoEM[1].

Many freeze substitution protocols have been developed and optimized for a wide range of specimen types since high pressure freezing (HPF) and automated freeze substitution (FS) systems were made available commercially. Most of these protocols require extended processing time from three to six days to ensure a slow warm up and a long FS period [2]. In a core imaging facility setting, the simultaneous processing of multiple and diverse samples usually occurs throughout a working week. In order to make the HPF and FS equipment available to more projects and to maximize the usage of a shared instrument, we experimented on a generalized FS protocol that is applicable to a wide range of specimens. Samples such as muscle, brain tissue, cultured cells and microorganisms were kept in liquid nitrogen after HPF and processed in batches in the FS system once a full load of samples has been accumulated.

Here we show examples of superior ultrastructure preservation for a variety of samples batchprocessed in our core facility (Figures 1 to 4). Our results highlight a practical approach to maximizing and optimizing the usage of a high functionality instrument in a multi-user core facility.

\section{References}

[1] P. Monaghan et al., J. Microscopy. 192 (1998) 248-58

[2] T.H.Giddings, J. Microscopy. 212 (1998) 53-61

[3] We thank Dr. Kim Rensing, Ms. Ann Korsen and Dr. Mark Kukucka for their assistance in conducting these experiments 


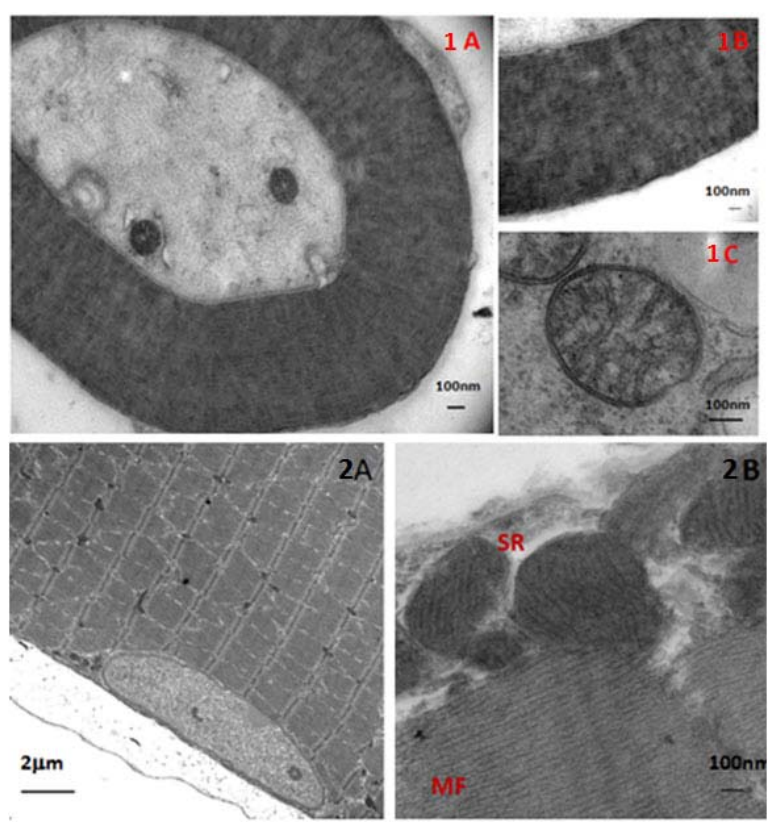

FIG. 1. Ultrastructure of mouse hippocampus processed by HPF, FS and embedding in Lowicryl at $-30^{\circ} \mathrm{C}(1 \mathrm{~A}-1 \mathrm{C})$. $1 \mathrm{~B}$ and $1 \mathrm{C}$ show high resolution details of the myelin structure and mitochondria in the hippocampus.

FIG. 2. Mouse tibialis anterior muscle prepared by HPF/FS cryo-preparation. The overall structure of muscle tissue is well preserved at $>20$ micron ice free preservation (2A). The ultrastructure of myofibrils (MF) and sarcoplasmic reticulum (SR) is also well preserved (2B).
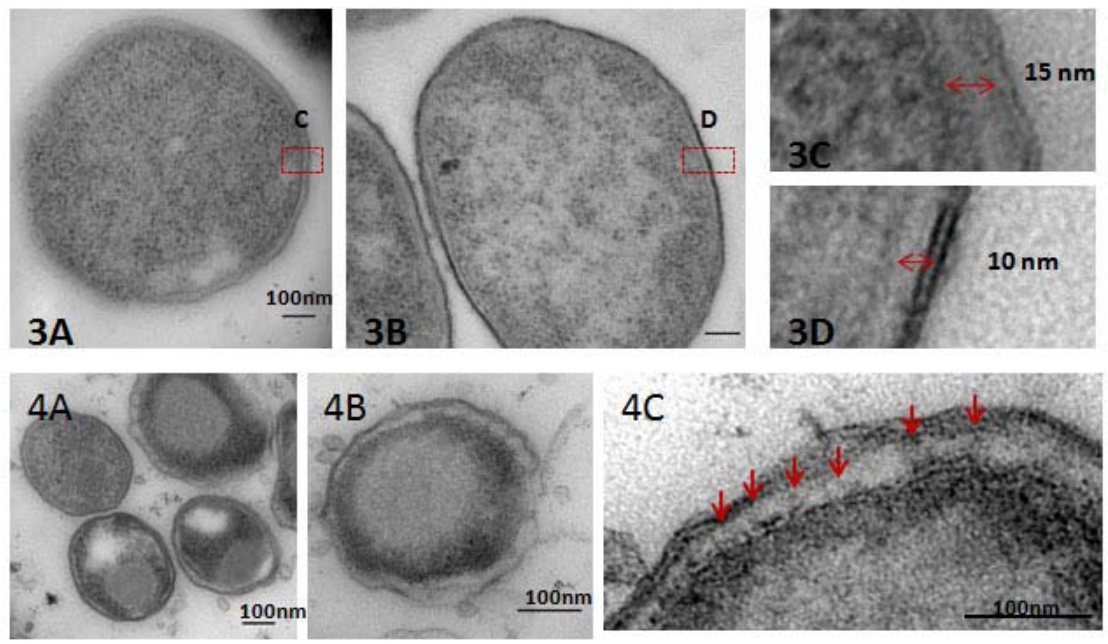

FIG. 3. Comparison of surface coat of enteropathogenic E. coli (EPEC) prepared by HPF/FS cryopreparation (3A) with conventional osmium and Spurr's embedding method (3B). The average width of the periplasmic space is $15 \mathrm{~nm}$ in HPF/FS specimens and $10 \mathrm{~nm}$ in osmicated and Spurrs' embedded specimens (3C \& 3D, respectively).

FIG. 4. Ultrastructural preservation of Chlamydia elementary bodies by HPF and FS. The chlamydial inner and outer membranes are well preserved and the lipid bilayer and periplasmic space are easily discernible (4B). Furthermore, there is clear indication of possible surface projection structures around the surface coat area (4C, red arrows). 